UDC 517.9

M. Mursaleen, A. K. Noman (Aligarh Muslim Univ., India)

\title{
ON SOME IMBEDDING RELATIONS BETWEEN CERTAIN SEQUENCE SPACES ${ }^{*}$ \\ ПРО ДЕЯКІ СПІВВІДНОШЕННЯ ВКЛАДЕННЯ МІЖ ПЕВНИМИ ПРОСТОРАМИ ПОСЛІДОВНОСТЕЙ
}

In the present paper, we introduce the sequence space $\ell_{p}^{\lambda}$ of non-absolute type which is a $p$-normed space and a $B K$-space in the cases of $0<p<1$ and $1 \leq p<\infty$, respectively. Further, we derive some imbedding relations and construct the basis for the space $\ell_{p}^{\lambda}$, where $1 \leq p<\infty$.

Введено поняття простору послідовностей $\ell_{p}^{\lambda}$ неабсолютного типу, який $є p$-нормованим простором і $B K$-простором у випадках $0<p<1$ і $1 \leq p<\infty$ відповідно. Крім того, отримано деякі співвідношення вкладення та побудовано базис для простору $\ell_{p}^{\lambda}$, де $1 \leq p<\infty$.

1. Introduction. By $w$, we denote the space of all complex valued sequences. Any vector subspace of $w$ is called a sequence space.

A sequence space $E$ with a linear topology is called a $K$-space provided each of the maps $p_{i}: E \rightarrow \mathbb{C}$ defined by $p_{i}(x)=x_{i}$ is continuous for all $i \in \mathbb{N}$; where $\mathbb{C}$ denotes the complex field and $\mathbb{N}=\{0,1,2, \ldots\}$. A $K$-space $E$ is called an $F K$-space provided $E$ is a complete linear metric space. An $F K$-space whose topology is normable is called a $B K$-space [2, p. 1451], that is, a $B K$-space is a Banach sequence space with continuous coordinates [11, p. 187].

We shall write $\ell_{\infty}, c$ and $c_{0}$ for the sequence spaces of all bounded, convergent and null sequences, respectively, which are $B K$-spaces with the usual sup-norm defined by

$$
\|x\|_{\ell_{\infty}}=\sup _{k}\left|x_{k}\right|
$$

where, here and in the sequel, the supremum $\sup _{k}$ is taken over all $k \in \mathbb{N}$. Also, by $\ell_{p}$, $0<p<\infty$, we denote the sequence space of all $p$-absolutely convergent series. It is known that the space $\ell_{p}$ is a complete $p$-normed space and a $B K$-space in the cases of $0<p<1$ and $1 \leq p<\infty$, respectively, with respect to the usual $p$-norm and $\ell_{p}$-norm defined by

$$
\|x\|_{\ell_{p}}=\sum_{k}\left|x_{k}\right|^{p}, \quad 0<p<1,
$$

and

$$
\|x\|_{\ell_{p}}=\left(\sum_{k}\left|x_{k}\right|^{p}\right)^{1 / p}, \quad 1 \leq p<\infty,
$$

respectively. For simplicity in notation, here and in what follows, the summation without limits runs from 0 to $\infty$.

Let $X$ and $Y$ be sequence spaces and $A=\left(a_{n k}\right)$ be an infinite matrix of complex numbers $a_{n k}$, where $n, k \in \mathbb{N}$. Then, we say that $A$ defines a matrix mapping from $X$ into $Y$, and we denote it by writing $A: X \rightarrow Y$, if for every sequence $x=\left(x_{k}\right) \in X$ the sequence $A x=\left\{A_{n}(x)\right\}$, the $A$-transform of $x$, exists and is in $Y$, where

\footnotetext{
* Research of the second author was supported by Department of Mathematics, Faculty of Education and Science, Al Baida University, Yemen.
} 


$$
A_{n}(x)=\sum_{k} a_{n k} x_{k}, \quad n \in \mathbb{N}
$$

By $(X: Y)$, we denote the class of all infinite matrices $A=\left(a_{n k}\right)$ such that $A: X \rightarrow$ $\rightarrow Y$. Thus, $A \in(X: Y)$ if and only if the series on the right-hand side of (1.1) converges for each $n \in \mathbb{N}$ and every $x \in X$, and $A x \in Y$ for all $x \in X$. A sequence $x$ is said to be $A$-summable to $l \in \mathbb{C}$ if $A x$ coverges to $l$ which is called the $A$-limit of $x$.

For a sequence space $X$, the matrix domain of an infinite matrix $A$ in $X$ is defined by

$$
X_{A}=\{x \in w: A x \in X\}
$$

which is a sequence space.

We shall write $e^{(k)}$ for the sequence whose only non-zero term is a 1 in the $k$ th place for each $k \in \mathbb{N}$.

The approach constructing a new sequence space by means of the matrix domain of a particular limitation method has recently been employed by several authors in many research papers (see, for example, $[1-7,12-15,17,18])$. The main purpose of this paper is to introduce the sequence space $\ell_{p}^{\lambda}$ of non-absolute type and is to derive some related results. Further, we establish some imbedding relations concerning the space $\ell_{p}^{\lambda}$, $0<p<\infty$. Finally, we construct the basis for the space $\ell_{p}^{\lambda}$, where $1 \leq p<\infty$.

2. The sequence space $\ell_{\boldsymbol{p}}^{\boldsymbol{\lambda}}$ of non-absolute type. Throughout this paper, let $\lambda=$ $=\left(\lambda_{k}\right)_{k \in \mathbb{N}}$ be a strictly increasing sequence of positive reals tending to $\infty$, that is

$$
0<\lambda_{0}<\lambda_{1}<\ldots \text { and } \lambda_{k} \rightarrow \infty \text { as } k \rightarrow \infty \text {. }
$$

By using the convention that any term with a negative subscript is equal to naught, we define the infinite matrix $\Lambda=\left(\lambda_{n k}\right)$ by

$$
\lambda_{n k}= \begin{cases}\frac{\lambda_{k}-\lambda_{k-1}}{\lambda_{n}}, & 0 \leq k \leq n, \\ 0, & k>n,\end{cases}
$$

for all $n, k \in \mathbb{N}$. Then, it is obvious by (2.2) that the matrix $\Lambda=\left(\lambda_{n k}\right)$ is a triangle, that is $\lambda_{n n} \neq 0$ and $\lambda_{n k}=0$ for all $k>n, n \in \mathbb{N}$. Further, by using (1.1), we have for every $x=\left(x_{k}\right) \in w$ that

$$
\Lambda_{n}(x)=\frac{1}{\lambda_{n}} \sum_{k=0}^{n}\left(\lambda_{k}-\lambda_{k-1}\right) x_{k}, \quad n \in \mathbb{N}
$$

Recently, Mursaleen and Noman [14] introduced the sequence spaces $c_{0}^{\lambda}, c^{\lambda}$ and $\ell_{\infty}^{\lambda}$ as follows:

$$
\begin{aligned}
& c_{0}^{\lambda}=\left\{x \in w: \lim _{n} \Lambda_{n}(x)=0\right\}, \\
& c^{\lambda}=\left\{x \in w: \lim _{n} \Lambda_{n}(x) \text { exists }\right\}
\end{aligned}
$$

and

$$
\ell_{\infty}^{\lambda}=\left\{x \in w: \sup _{n}\left|\Lambda_{n}(x)\right|<\infty\right\}
$$


Moreover, it has been shown that the inclusions $c_{0} \subset c_{0}^{\lambda}, c \subset c^{\lambda}$ and $\ell_{\infty} \subset \ell_{\infty}^{\lambda}$ hold. We refer the reader to [14] for relevant terminology.

Now, as a natural continuation of the above spaces, we define $\ell_{p}^{\lambda}$ as the set of all sequences whose $\Lambda$-transforms are in the space $\ell_{p}, 0<p<\infty$; that is

$$
\ell_{p}^{\lambda}=\left\{x \in w: \sum_{n}\left|\Lambda_{n}(x)\right|^{p}<\infty\right\}, \quad 0<p<\infty .
$$

With the notation of (1.2), we may redefine the space $\ell_{p}^{\lambda}, 0<p<\infty$ as the matrix domain of the triangle $\Lambda$ in the space $\ell_{p}$. This can be written as follows:

$$
\ell_{p}^{\lambda}=\left(\ell_{p}\right)_{\Lambda}, \quad 0<p<\infty
$$

It is trivial that $\ell_{p}^{\lambda}, 0<p<\infty$, is a linear space with the coordinatewise addition and scalar multiplication. Further, it follows by (2.4) that the space $\ell_{p}^{\lambda}, 0<p<1$, becomes a $p$-normed space with the following $p$-norm:

$$
\|x\|_{\ell_{p}^{\lambda}}=\|\Lambda(x)\|_{\ell_{p}}=\sum_{n}\left|\Lambda_{n}(x)\right|^{p}, \quad 0<p<1 .
$$

Moreover, since the matrix $\Lambda$ is a triangle, we have the following result which is essential in the text.

Theorem 2.1. The sequence space $\ell_{p}^{\lambda}, 1 \leq p<\infty$, is a BK-space with the norm given by

$$
\|x\|_{\ell_{p}^{\lambda}}=\|\Lambda(x)\|_{\ell_{p}}=\left(\sum_{n}\left|\Lambda_{n}(x)\right|^{p}\right)^{1 / p}, \quad 1 \leq p<\infty .
$$

Proof. Since (2.4) holds and $\ell_{p}, 1 \leq p<\infty$, is a $B K$-space with the $\ell_{p}$-norm (see $[10$, p. 218]), this result is immediate by Theorem 4.3.12 of Wilansky [19, p. 63].

Remark 2.1. One can easily check that the absolute property does not hold on the space $\ell_{p}^{\lambda}, 0<p<\infty$, that is $\|x\|_{\ell_{p}^{\lambda}} \neq\|\mid x\|_{\ell_{p}^{\lambda}}$ for at least one sequence $x \in \ell_{p}^{\lambda}$. This tells us that $\ell_{p}^{\lambda}$ is a sequence space of non-absolute type, where $|x|=\left(\left|x_{k}\right|\right)$.

Theorem 2.2. The sequence space $\ell_{p}^{\lambda}$ of non-absolute type is linear isometric to the space $\ell_{p}$, where $0<p<\infty$.

Proof. To prove this, we should show the existence of a linear isometry between the spaces $\ell_{p}^{\lambda}$ and $\ell_{p}$, where $0<p<\infty$. For this, let us consider the transformation $T$ defined, with the notation of (2.3), from $\ell_{p}^{\lambda}$ to $\ell_{p}$ by $x \longmapsto \Lambda(x)=T x$. Then $T x=\Lambda(x) \in \ell_{p}$ for every $x \in \ell_{p}^{\lambda}$. Also, the linearity of $T$ is trivial. Further, it is easiy to see that $x=0$ whenever $T x=0$ and hence $T$ is injective.

Furthermore, for any given $y=\left(y_{k}\right) \in \ell_{p}$, we define the sequence $x=\left(x_{k}\right)$ by

$$
x_{k}=\frac{\lambda_{k} y_{k}-\lambda_{k-1} y_{k-1}}{\lambda_{k}-\lambda_{k-1}}, \quad k \in \mathbb{N} .
$$

Then, we have for every $n \in \mathbb{N}$ that

$$
\Lambda_{n}(x)=\frac{1}{\lambda_{n}} \sum_{k=0}^{n}\left(\lambda_{k}-\lambda_{k-1}\right) x_{k}=\frac{1}{\lambda_{n}} \sum_{k=0}^{n}\left(\lambda_{k} y_{k}-\lambda_{k-1} y_{k-1}\right)=y_{n} .
$$


This shows that $\Lambda(x)=y$ and since $y \in \ell_{p}$, we obtain that $\Lambda(x) \in \ell_{p}$. Thus, we deduce that $x \in \ell_{p}^{\lambda}$ and $T x=y$. Hence, the operator $T$ is surjective.

Moreover, let $x \in \ell_{p}^{\lambda}$ be given. Then, we have that

$$
\|T x\|_{\ell_{p}}=\|\Lambda(x)\|_{\ell_{p}}=\|x\|_{\ell_{p}^{\lambda}}
$$

and hence $T$ is an isometry. Consequently, the spaces $\ell_{p}^{\lambda}$ and $\ell_{p}$ are linear isometric for $0<p<\infty$.

Theorem 2.2 is proved.

Finally, we know that the space $\ell_{2}$ is the only Hilbert space among the Banach spaces $\ell_{p}, 1 \leq p<\infty$. Thus, we conclude this section with the following corollary which is immediate by Theorems 2.1 and 2.2 .

Corollary 2.1. Except the case $p=2$, the space $\ell_{p}^{\lambda}$ is not an inner product space, hence not a Hilbert space for $1 \leq p<\infty$.

3. Some imbedding relations. In the present section, we establish some imbedding relations concerning the space $\ell_{p}^{\lambda}, 0<p<\infty$. We essentially characterize the case in which the imbedding $\ell_{p} \subset \ell_{p}^{\lambda}$ holds for $1 \leq p<\infty$.

The notion of imbedded Banach spaces can be found in [9] (Chapter I) and it can be given as follows:

Let $X$ and $Y$ be Banach spaces. Then, we say that $X$ is imbedded in $Y$ if the following conditions are satisfied:

(i) $x \in X$ implies $x \in Y$, that is, the space $Y$ includes $X$.

(ii) The space $Y$ includes a vector space structure on $X$ coinciding with the structure of $X$.

(iii) There exists a constant $C>0$ such that $\|x\|_{Y} \leq C\|x\|_{X}$ for all $x \in X$.

In what follows, we shall denote the imbedding of $X$ in $Y$ by $X \subset Y$, assuming that the symbol $\subset$ means not only the set-theoretic inclusion, but imbedding have the properties (ii) and (iii). Further, we say that the imbedding $X \subset Y$ strictly holds if the space $Y$ strictly includes $X$.

Since any two sequence spaces have the same vector space structure, the condition (ii) is redundant when $X$ and $Y$ are $B K$-spaces.

Now, we may begin with the following basic result:

Theorem 3.1. If $0<p<s<\infty$, then the imbedding $\ell_{p}^{\lambda} \subset \ell_{s}^{\lambda}$ strictly holds.

Proof. Since the space $\ell_{s}$ strictly includes $\ell_{p}$, the space $\ell_{s}^{\lambda}$ strictly includes $\ell_{p}^{\lambda}$. Therefore, this result is immediate by the fact that the topology of the space $\ell_{p}^{\lambda}$ is stronger than the topology of $\ell_{s}^{\lambda}$, that is

$$
\|x\|_{\ell_{s}^{\lambda}}=\|\Lambda(x)\|_{\ell_{s}} \leq\|\Lambda(x)\|_{\ell_{p}}=\|x\|_{\ell_{p}^{\lambda}}
$$

for all $x \in \ell_{p}^{\lambda}$, where $0<p<s<\infty$.

Theorem 3.1 is proved.

Although the imbeddings $c_{0} \subset c_{0}^{\lambda}, c \subset c^{\lambda}$ and $\ell_{\infty} \subset \ell_{\infty}^{\lambda}$ always holds, the space $\ell_{p}$ may not be included in $\ell_{p}^{\lambda}$ for $0<p<\infty$. This will be shown in the following lemma in which we write $\frac{1}{\lambda}=\left(\frac{1}{\lambda_{k}}\right)$.

Lemma 3.1. Let $0<p<\infty$. Then, the spaces $\ell_{p}$ and $\ell_{p}^{\lambda}$ overlap. Further, if $\frac{1}{\lambda} \notin \ell_{p}$ then neither of the spaces $\ell_{p}$ and $\ell_{p}^{\lambda}$ includes the other one. 
Proof. Obviously, the spaces $\ell_{p}$ and $\ell_{p}^{\lambda}$ always overlap, since the sequence $\left(\lambda_{1}-\right.$ $\left.-\lambda_{0},-\lambda_{0}, 0,0, \ldots\right)$ belongs to both spaces $\ell_{p}$ and $\ell_{p}^{\lambda}$ for $0<p<\infty$.

Suppose now that $\frac{1}{\lambda} \notin \ell_{p}, 0<p<\infty$, and consider the sequence $x=e^{(0)}=$ $=(1,0,0, \ldots) \in \ell_{p}$. Then, by using (2.3), we have for every $n \in \mathbb{N}$ that

$$
\Lambda_{n}(x)=\frac{1}{\lambda_{n}} \sum_{k=0}^{n}\left(\lambda_{k}-\lambda_{k-1}\right) e_{k}^{(0)}=\frac{\lambda_{0}}{\lambda_{n}} .
$$

Thus, we obtain that

$$
\sum_{n}\left|\Lambda_{n}(x)\right|^{p}=\lambda_{0}^{p} \sum_{n} \frac{1}{\lambda_{n}^{p}}
$$

which shows that $\Lambda(x) \notin \ell_{p}$ and hence $x \notin \ell_{p}^{\lambda}$. Thus, the sequence $x$ is in $\ell_{p}$ but not in $\ell_{p}^{\lambda}$. Hence, the space $\ell_{p}^{\lambda}$ does not include $\ell_{p}$ when $\frac{1}{\lambda} \notin \ell_{p}$, where $0<p<\infty$.

On the other hand, let $1 \leq p<\infty$ and define the sequence $y=\left(y_{k}\right)$ by

$$
y_{k}=\left\{\begin{array}{ll}
\frac{1}{\lambda_{k}}, & k \text { is even, } \\
-\frac{1}{\lambda_{k-1}}\left(\frac{\lambda_{k-1}-\lambda_{k-2}}{\lambda_{k}-\lambda_{k-1}}\right), & k \text { is odd, }
\end{array} \quad k \in \mathbb{N} .\right.
$$

Then $y \notin \ell_{p}$, since $\frac{1}{\lambda} \notin \ell_{p}$. Besides, we have for every $n \in \mathbb{N}$ that

$$
\Lambda_{n}(y)= \begin{cases}\frac{1}{\lambda_{n}}\left(\frac{\lambda_{n}-\lambda_{n-1}}{\lambda_{n}}\right), & n \text { is even } \\ 0, & n \text { is odd }\end{cases}
$$

Thus, we obtain that

$$
\begin{aligned}
\sum_{n}\left|\Lambda_{n}(y)\right|^{p} & =\sum_{n}\left|\Lambda_{2 n}(y)\right|^{p}=\sum_{n} \frac{1}{\lambda_{2 n}^{p}}\left(\frac{\lambda_{2 n}-\lambda_{2 n-1}}{\lambda_{2 n}}\right)^{p} \leq \\
\leq & \frac{1}{\lambda_{0}^{p}}+\sum_{n=1}^{\infty} \frac{1}{\lambda_{2 n-2}^{p}}\left(\frac{\lambda_{2 n}-\lambda_{2 n-2}}{\lambda_{2 n}}\right)^{p} \leq \\
\leq & \frac{1}{\lambda_{0}^{p}}+\sum_{n=1}^{\infty} \frac{1}{\lambda_{2 n-2}^{p}}\left(\frac{\lambda_{2 n}^{p}-\lambda_{2 n-2}^{p}}{\lambda_{2 n}^{p}}\right)= \\
= & \frac{1}{\lambda_{0}^{p}}+\sum_{n=1}^{\infty}\left(\frac{1}{\lambda_{2 n-2}^{p}}-\frac{1}{\lambda_{2 n}^{p}}\right)=\frac{2}{\lambda_{0}^{p}}<\infty .
\end{aligned}
$$

This shows that $\Lambda(y) \in \ell_{p}$ and hence $y \in \ell_{p}^{\lambda}$. Thus, the sequence $y$ is in $\ell_{p}^{\lambda}$ but not in $\ell_{p}$, where $1 \leq p<\infty$.

Similarly, one can construct a sequence belonging to the set $\ell_{p}^{\lambda} \backslash \ell_{p}$ for $0<p<1$. Therefore, the space $\ell_{p}$ also does not include $\ell_{p}^{\lambda}$ when $\frac{1}{\lambda} \notin \ell_{p}$ for $0<p<\infty$.

Lemma 3.1 is proved.

As an immediate consequence of Lemma 3.1, we have the following lemma. 
Lemma 3.2. If the imbedding $\ell_{p} \subset \ell_{p}^{\lambda}$ holds, then $\frac{1}{\lambda} \in \ell_{p}$, where $0<p<\infty$.

Proof. Suppose that the imbedding $\ell_{p} \subset \ell_{p}^{\lambda}$ holds, where $0<p<\infty$, and consider the sequence $x=e^{(0)}=(1,0,0, \ldots) \in \ell_{p}$. Then $x \in \ell_{p}^{\lambda}$ and hence $\Lambda(x) \in \ell_{p}$. Thus, we obtain that

$$
\lambda_{0}^{p} \sum_{n}\left(\frac{1}{\lambda_{n}}\right)^{p}=\sum_{n}\left|\Lambda_{n}(x)\right|^{p}<\infty
$$

which shows that $\frac{1}{\lambda} \in \ell_{p}$.

Lemma 3.2 is proved.

We shall later show that the condition $\frac{1}{\lambda} \in \ell_{p}$ is not only necessary but also sufficient for the imbedding $\ell_{p} \subset \ell_{p}^{\lambda}$ to be held, where $1 \leq p<\infty$. Before that, by taking into account the definition of the sequence $\lambda=\left(\lambda_{k}\right)$ given by (2.1), we find that

$$
0<\frac{\lambda_{k}-\lambda_{k-1}}{\lambda_{n}}<1, \quad 0 \leq k \leq n
$$

for all $n, k \in \mathbb{N}$ with $n+k>0$. Furthermore, if $\frac{1}{\lambda} \in \ell_{1}$ then we have the following lemma which is easy to prove.

Lemma 3.3. If $\frac{1}{\lambda} \in \ell_{1}$, then

$$
\sup _{k}\left(\lambda_{k}-\lambda_{k-1}\right) \sum_{n=k}^{\infty} \frac{1}{\lambda_{n}}<\infty .
$$

Now, we prove the following:

Theorem 3.2. The imbedding $\ell_{1} \subset \ell_{1}^{\lambda}$ holds if and only if $\frac{1}{\lambda} \in \ell_{1}$.

Proof. The necessity is immediate by Lemma 3.2.

Conversely, suppose that $\frac{1}{\lambda} \in \ell_{1}$. Then, it follows by Lemma 3.3 that

$$
M=\sup _{k}\left(\lambda_{k}-\lambda_{k-1}\right) \sum_{n=k}^{\infty} \frac{1}{\lambda_{n}}<\infty .
$$

Therefore, we have for every $x=\left(x_{k}\right) \in \ell_{1}$ that

$$
\begin{aligned}
& \|x\|_{\ell_{1}^{\lambda}}=\sum_{n}\left|\Lambda_{n}(x)\right| \leq \sum_{n=0}^{\infty} \frac{1}{\lambda_{n}} \sum_{k=0}^{n}\left(\lambda_{k}-\lambda_{k-1}\right)\left|x_{k}\right|= \\
& =\sum_{k=0}^{\infty}\left|x_{k}\right|\left(\lambda_{k}-\lambda_{k-1}\right) \sum_{n=k}^{\infty} \frac{1}{\lambda_{n}} \leq M \sum_{k=0}^{\infty}\left|x_{k}\right|=M\|x\|_{\ell_{1}} .
\end{aligned}
$$

This also shows that the space $\ell_{1}^{\lambda}$ includes $\ell_{1}$. Hence, the imbedding $\ell_{1} \subset \ell_{1}^{\lambda}$ holds which concludes the proof.

Corollary 3.1. If $\frac{1}{\lambda} \in \ell_{1}$, then the imbedding $\ell_{p} \subset \ell_{p}^{\lambda}$ holds for $1 \leq p<\infty$.

Proof. The imbedding trivially holds for $p=1$ by Theorem 3.2, above. Thus, let $1<p<\infty$ and take any $x \in \ell_{p}$. Then $|x|^{p} \in \ell_{1}$ and hence $|x|^{p} \in \ell_{1}^{\lambda}$ by Theorem 3.2 which implies that $x \in \ell_{p}^{\lambda}$. This shows that the space $\ell_{p}$ is included in $\ell_{p}^{\lambda}$. 
Further, let $x=\left(x_{k}\right) \in \ell_{p}$ be given. Then, for every $n \in \mathbb{N}$, we obtain by applying the Hölder's inequality that

$$
\begin{gathered}
\left|\Lambda_{n}(x)\right|^{p} \leq\left[\sum_{k=0}^{n}\left(\frac{\lambda_{k}-\lambda_{k-1}}{\lambda_{n}}\right)\left|x_{k}\right|\right]^{p} \leq \\
\leq\left[\sum_{k=0}^{n}\left(\frac{\lambda_{k}-\lambda_{k-1}}{\lambda_{n}}\right)\left|x_{k}\right|^{p}\right]\left[\sum_{k=0}^{n} \frac{\lambda_{k}-\lambda_{k-1}}{\lambda_{n}}\right]^{p-1}=\frac{1}{\lambda_{n}} \sum_{k=0}^{n}\left(\lambda_{k}-\lambda_{k-1}\right)\left|x_{k}\right|^{p} .
\end{gathered}
$$

Thus, we derive that

$$
\begin{gathered}
\|x\|_{\ell_{p}^{\lambda}}^{p}=\sum_{n}\left|\Lambda_{n}(x)\right|^{p} \leq \sum_{n=0}^{\infty} \frac{1}{\lambda_{n}} \sum_{k=0}^{n}\left(\lambda_{k}-\lambda_{k-1}\right)\left|x_{k}\right|^{p}= \\
=\sum_{k=0}^{\infty}\left|x_{k}\right|^{p}\left(\lambda_{k}-\lambda_{k-1}\right) \sum_{n=k}^{\infty} \frac{1}{\lambda_{n}} \leq M \sum_{k=0}^{\infty}\left|x_{k}\right|^{p}=M\|x\|_{\ell_{p}}^{p},
\end{gathered}
$$

where $M=\sup _{k}\left[\left(\lambda_{k}-\lambda_{k-1}\right) \sum_{n=k}^{\infty} \frac{1}{\lambda_{n}}\right]<\infty$ by Lemma 3.3. Hence, the imbedding $\ell_{p} \subset \ell_{p}^{\lambda}$ also holds for $1<p<\infty$.

Corollary 3.1 is proved.

Now, as a generalization of Theorem 3.2, the following theorem shows the necessity and sufficiency of the condition $\frac{1}{\lambda} \in \ell_{p}$ for the imbedding $\ell_{p} \subset \ell_{p}^{\lambda}$ to be held, where $1 \leq p<\infty$.

Theorem 3.3. The imbedding $\ell_{p} \subset \ell_{p}^{\lambda}$ holds if and only if $\frac{1}{\lambda} \in \ell_{p}$, where $1 \leq p<$ $<\infty$.

Proof. The necessity is trivial by Lemma 3.2. Thus, we turn to the sufficiency. For this, suppose that $\frac{1}{\lambda} \in \ell_{p}$, where $1 \leq p<\infty$. Then $\frac{1}{\lambda^{p}}=\left(\frac{1}{\lambda_{k}^{p}}\right) \in \ell_{1}$. Therefore, it follows by Lemma 3.3 that

$$
\sup _{k}\left(\lambda_{k}-\lambda_{k-1}\right)^{p} \sum_{n=k}^{\infty} \frac{1}{\lambda_{n}^{p}} \leq \sup _{k}\left(\lambda_{k}^{p}-\lambda_{k-1}^{p}\right) \sum_{n=k}^{\infty} \frac{1}{\lambda_{n}^{p}}<\infty .
$$

Further, we have for every fixed $k \in \mathbb{N}$ that

$$
\Lambda_{n}\left(e^{(k)}\right)=\left\{\begin{array}{ll}
\frac{\lambda_{k}-\lambda_{k-1}}{\lambda_{n}}, & 0 \leq k \leq n, \\
0, & k>n,
\end{array} \quad n \in \mathbb{N} .\right.
$$

Thus, we obtain that

$$
\left\|e^{(k)}\right\|_{\ell_{p}^{\lambda}}^{p}=\left(\lambda_{k}-\lambda_{k-1}\right)^{p} \sum_{n=k}^{\infty} \frac{1}{\lambda_{n}^{p}}<\infty, \quad k \in \mathbb{N}
$$

which yields that $e^{(k)} \in \ell_{p}^{\lambda}$ for every $k \in \mathbb{N}$, i.e., every basis element of the space $\ell_{p}$ is in $\ell_{p}^{\lambda}$. This shows that the space $\ell_{p}^{\lambda}$ contains the Schauder basis for the space $\ell_{p}$ such 
that

$$
\sup _{k}\left\|e^{(k)}\right\|_{\ell_{p}^{\lambda}}<\infty .
$$

Therefore, we deduce that the space $\ell_{p}^{\lambda}$ includes $\ell_{p}$. Moreover, by using the same technique used in the proof of Corollary 3.1, it can similarly be shown that the topology of the space $\ell_{p}$ is stronger than the topology of $\ell_{p}^{\lambda}$. Hence, the imbedding $\ell_{p} \subset \ell_{p}^{\lambda}$ holds, where $1 \leq p<\infty$.

Theorem 3.3 is proved.

Now, in the following example, we give an important particular case of the space $\ell_{p}^{\lambda}$, where $1 \leq p<\infty$.

Example 3.1. Consider the particular case of the sequence $\lambda=\left(\lambda_{k}\right)$ given by $\lambda_{k}=k+1$ for all $k \in \mathbb{N}$. Then $\frac{1}{\lambda} \notin \ell_{1}$ and hence $\ell_{1}$ is not included in $\ell_{1}^{\lambda}$ by Lemma 3.1.

On the other hand, we have $\frac{1}{\lambda} \in \ell_{p}$ for $1<p<\infty$ and so $\ell_{p}$ is included in $\ell_{p}^{\lambda}$. Further, by applying the well-known inequality (see [8, p. 239])

$$
\sum_{n}\left(\sum_{k=0}^{n} \frac{\left|x_{k}\right|}{n+1}\right)^{p}<\left(\frac{p}{p-1}\right)^{p} \sum_{n}\left|x_{n}\right|^{p}, \quad 1<p<\infty,
$$

we immediately obtain that

$$
\|x\|_{\ell_{p}^{\lambda}}<\frac{p}{p-1}\|x\|_{\ell_{p}}, \quad 1<p<\infty
$$

for all $x \in \ell_{p}$. This shows that the imbedding $\ell_{p} \subset \ell_{p}^{\lambda}$ holds for $1<p<\infty$. Moreover, this imbedding is strict. For example, the sequence $y=\left\{(-1)^{k}\right\}_{k \in \mathbb{N}}$ is not in $\ell_{p}$ but in $\ell_{p}^{\lambda}$, since

$$
\sum_{n}\left|\Lambda_{n}(y)\right|^{p}=\sum_{n}\left|\frac{1}{n+1} \sum_{k=0}^{n}(-1)^{k}\right|^{p}=\sum_{n} \frac{1}{(2 n+1)^{p}}<\infty, \quad 1<p<\infty .
$$

Remark 3.1. In the special case $\lambda_{k}=k+1(k \in \mathbb{N})$ given in Example 3.1, we may note that the space $\ell_{p}^{\lambda}$ is reduced to the Cesàro sequence space $X_{p}$ of non-absolute type, where $1 \leq p<\infty$ (see $[16,17])$.

Now, let $x=\left(x_{k}\right)$ be a null sequence of positive reals, that is

$$
x_{k}>0 \text { for all } k \in \mathbb{N} \text { and } x_{k} \rightarrow 0 \text { as } k \rightarrow \infty .
$$

Then, as is easy to see, for every positive integer $m$ there is a subsequence $\left\{x_{k_{r}}\right\}_{r \in \mathbb{N}}$ of the sequence $x$ such that

$$
x_{k_{r}}=O\left(\frac{1}{(r+1)^{m+1}}\right) .
$$

Further, this subsequence can be chosen such that $k_{r+1}-k_{r} \geq 2$ for all $r \in \mathbb{N}$.

In general, if $x=\left(x_{k}\right)$ is a sequence of positive reals such that liminf $x_{k}=0$, then there is a subsequence $x^{\prime}=\left\{x_{k_{r}^{\prime}}\right\}_{r \in \mathbb{N}}$ of the sequence $x$ such that $\lim _{r} x_{k_{r}^{\prime}}=0$. Thus $x^{\prime}$ is a null sequence of positive reals. Hence, as we have seen above, for every positive integer $m$ there is a subsequence $\left\{x_{k_{r}}\right\}_{r \in \mathbb{N}}$ of the sequence $x^{\prime}$ and hence of the sequence $x$ such that $k_{r+1}-k_{r} \geq 2$ for all $r \in \mathbb{N}$ and 


$$
x_{k_{r}}=O\left(\frac{1}{(r+1)^{m+1}}\right),
$$

where $k_{r}=k_{\theta(r)}^{\prime}$ and $\theta: \mathbb{N} \rightarrow \mathbb{N}$ is a suitable increasing function. Thus, we obtain that

$$
(r+1) x_{k_{r}}=O\left(\frac{1}{(r+1)^{m}}\right) .
$$

Now, let $0<p<\infty$. Then, we can choose a positive integer $m$ such that $m p>1$. In this situation, the sequence $\left\{(r+1) x_{k_{r}}\right\}_{r \in \mathbb{N}}$ is in the space $\ell_{p}$.

Obviously, we observe that the subsequence $\left\{x_{k_{r}}\right\}_{r \in \mathbb{N}}$ depends on the positive integer $m$ which is, in turn, depending on $p$. Thus, our subsequence depends on $p$.

Hence, from the above discussion, we conclude the following result:

Lemma 3.4. Let $x=\left(x_{k}\right)$ be a sequence of positive reals such that $\lim \inf x_{k}=0$. Then, for every positive number $p \in(0, \infty)$ there is a subsequence $x^{(p)}=\left\{x_{k_{r}}\right\}_{r \in \mathbb{N}}$ of $x$, depending on $p$, such that $k_{r+1}-k_{r} \geq 2$ for all $r \in \mathbb{N}$ and $\sum_{r}\left|(r+1) x_{k_{r}}\right|^{p}<\infty$.

Moreover, we have the following two lemmas (see [14]) which are needed in the sequel.

Lemma 3.5. For any sequence $x=\left(x_{k}\right) \in w$, the equalities

$$
S_{n}(x)=x_{n}-\Lambda_{n}(x), \quad n \in \mathbb{N},
$$

and

$$
S_{n}(x)=\frac{\lambda_{n-1}}{\lambda_{n}-\lambda_{n-1}}\left[\Lambda_{n}(x)-\Lambda_{n-1}(x)\right], \quad n \in \mathbb{N},
$$

hold, where the sequence $S(x)=\left\{S_{n}(x)\right\}$ is defined by

$$
S_{0}(x)=0 \text { and } S_{n}(x)=\frac{1}{\lambda_{n}} \sum_{k=1}^{n} \lambda_{k-1}\left(x_{k}-x_{k-1}\right) \text { for } n \geq 1 .
$$

Lemma 3.6. For any sequence $\lambda=\left(\lambda_{k}\right)$ satisfying (2.1), the sequence $\left\{\frac{\lambda_{k}}{\lambda_{k}-\lambda_{k-1}}\right\}_{k \in \mathbb{N}}$ is bounded if and only if $\liminf \frac{\lambda_{k+1}}{\lambda_{k}}>1$, and is unbounded if and only if lim inf $\frac{\lambda_{k+1}}{\lambda_{k}}=1$.

Now, we know by Theorem 3.3 that the imbedding $\ell_{p} \subset \ell_{p}^{\lambda}$ holds whenever $\frac{1}{\lambda} \in \ell_{p}$, $1 \leq p<\infty$. More precisely, the following theorem gives the necessary and sufficient conditions for this imbedding to be strict.

Theorem 3.4. Let $1 \leq p<\infty$. Then, the imbedding $\ell_{p} \subset \ell_{p}^{\lambda}$ strictly holds if and only if $\frac{1}{\lambda} \in \ell_{p}$ and $\lim \inf \frac{\lambda_{n+1}}{\lambda_{n}}=1$.

Proof. Suppose that the imbedding $\ell_{p} \subset \ell_{p}^{\lambda}$ is strict, where $1 \leq p<\infty$. Then, the necessity of the first condition is immediate by Theorem 3.3. Further, since $\ell_{p}^{\lambda}$ strictly includes $\ell_{p}$, there is a sequence $x \in \ell_{p}^{\lambda}$ such that $x \notin \ell_{p}$, that is $\Lambda(x) \in \ell_{p}$ while $x \notin \ell_{p}$. Thus, we obtain by (3.1) of Lemma 3.5 that $S(x)=\left\{S_{n}(x)\right\} \notin \ell_{p}$. Moreover, since $\Lambda(x) \in \ell_{p}$, we have $\sum_{n}\left|\Lambda_{n}(x)\right|^{p}<\infty$ and hence $\sum_{n}\left|\Lambda_{n}(x)-\Lambda_{n-1}(x)\right|^{p}<\infty$ by applying the Minkowski's inequality. This means that $\left\{\Lambda_{n}(x)-\Lambda_{n-1}(x)\right\} \in \ell_{p}$. Thus, by combining this with the fact that $\left\{S_{n}(x)\right\} \notin \ell_{p}$, it follows by (3.2) that the 
sequence $\left\{\frac{\lambda_{n-1}}{\lambda_{n}-\lambda_{n-1}}\right\}$ is unbounded and hence $\left\{\frac{\lambda_{n}}{\lambda_{n}-\lambda_{n-1}}\right\} \notin \ell_{\infty}$. This leads us with Lemma 3.6 to the necessity of the second condition.

Conversely, since $\frac{1}{\lambda} \in \ell_{p}$, we have by Theorem 3.3 that the imbedding $\ell_{p} \subset \ell_{p}^{\lambda}$ holds. Further, since $\lim \inf \frac{\lambda_{k+1}}{\lambda_{k}}=1$, we obtain by Lemma 3.6 that

$$
\liminf \frac{\lambda_{k}-\lambda_{k-1}}{\lambda_{k}}=0
$$

Thus, it follows by Lemma 3.4 that there is a subsequence $\lambda^{(p)}=\left\{\lambda_{k_{r}}\right\}_{r \in \mathbb{N}}$ of the sequence $\lambda=\left(\lambda_{k}\right)$, depending on $p$, such that $k_{r+1}-k_{r} \geq 2$ for all $r \in \mathbb{N}$ and

$$
\sum_{r}\left|(r+1)\left(\frac{\lambda_{k_{r}}-\lambda_{k_{r}-1}}{\lambda_{k_{r}}}\right)\right|^{p}<\infty .
$$

Let us now define the sequence $y=\left(y_{k}\right)$ for every $k \in \mathbb{N}$ by

$$
y_{k}= \begin{cases}r+1, & k=k_{r}, \\ -(r+1)\left(\frac{\lambda_{k-1}-\lambda_{k-2}}{\lambda_{k}-\lambda_{k-1}}\right), & k=k_{r}+1, \quad r \in \mathbb{N}, \\ 0, & \text { otherwise. }\end{cases}
$$

Then, it is clear that $y \notin \ell_{p}$. Moreover, we have for every $n \in \mathbb{N}$ that

$$
\Lambda_{n}(y)= \begin{cases}(r+1)\left(\frac{\lambda_{n}-\lambda_{n-1}}{\lambda_{n}}\right), & n=k_{r}, \\ 0, & n \neq k_{r},\end{cases}
$$

This and (3.3) imply that $\Lambda(y) \in \ell_{p}$ and hence $y \in \ell_{p}^{\lambda}$. Thus, the sequence $y$ is in $\ell_{p}^{\lambda}$ but not in $\ell_{p}$. Therefore, the imbedding $\ell_{p} \subset \ell_{p}^{\lambda}$ strictly holds, where $1 \leq p<\infty$.

Theorem 3.4 is proved.

As an immediate consequence of Theorem 3.4, we have the following result:

Theorem 3.5. The equality $\ell_{p}^{\lambda}=\ell_{p}$ holds if and only if $\lim \inf \frac{\lambda_{n+1}}{\lambda_{n}}>1$, where $1 \leq p<\infty$.

Proof. The necessity is immediate by Theorems 3.3 and 3.4. For, if the equality holds then $\ell_{p}$ is imbedded in $\ell_{p}^{\lambda}$ and hence $\frac{1}{\lambda} \in \ell_{p}$ by Theorem 3.3. Further, since the imbedding $\ell_{p} \subset \ell_{p}^{\lambda}$ cannot be strict, we have by Theorem 3.4 that $\lim \inf \frac{\lambda_{n+1}}{\lambda_{n}} \neq 1$ and hence $\liminf \frac{\lambda_{n+1}}{\lambda_{n}}>1$.

Conversely, suppose that $\lim \inf \frac{\lambda_{n+1}}{\lambda_{n}}>1$. Then, there exists a constant $a>1$ such that $\frac{\lambda_{n+1}}{\lambda_{n}} \geq a$ and hence $\lambda_{n} \geq \lambda_{0} a^{n}$ for all $n \in \mathbb{N}$. This shows that $\frac{1}{\lambda} \in \ell_{1}$ which leads us with Corollary 3.1 to the consequence that the imbedding $\ell_{p} \subset \ell_{p}^{\lambda}$ holds and hence $\ell_{p}$ is included in $\ell_{p}^{\lambda}$, where $1 \leq p<\infty$. 
On the other hand, by using Lemma 3.6, we have the bounded sequence $\left\{\frac{\lambda_{n}}{\lambda_{n}-\lambda_{n-1}}\right\}$ and hence $\left\{\frac{\lambda_{n-1}}{\lambda_{n}-\lambda_{n-1}}\right\} \in \ell_{\infty}$.

Now, let $x \in \ell_{p}^{\lambda}$. Then $\Lambda(x)=\left\{\Lambda_{n}(x)\right\} \in \ell_{p}$ and hence $\left\{\Lambda_{n}(x)-\Lambda_{n-1}(x)\right\} \in \ell_{p}$. Thus, we obtain by (3.2) that $S(x)=\left\{S_{n}(x)\right\} \in \ell_{p}$. Therefore, it follows by (3.1) that $x=S(x)+\Lambda(x) \in \ell_{p}$. This shows that $x \in \ell_{p}$ for all $x \in \ell_{p}^{\lambda}$ and hence $\ell_{p}^{\lambda}$ is also included in $\ell_{p}$. Consequently, the equality $\ell_{p}^{\lambda}=\ell_{p}$ holds, where $1 \leq p<\infty$.

Theorem 3.5 is proved.

Finally, we conclude this section by the following corollary:

Corollary 3.2. Although the spaces $\ell_{p}^{\lambda}, c_{0}, c$ and $\ell_{\infty}$ overlap, the space $\ell_{p}^{\lambda}$ does not include any of the other spaces. Further, if $\lim \inf \frac{\lambda_{n+1}}{\lambda_{n}}=1$ then none of the spaces $c_{0}$, c or $\ell_{\infty}$ includes the space $\ell_{p}^{\lambda}$, where $0<p<\infty$.

Proof. Let $0<p<\infty$. Then, it is obvious by Lemma 3.1 that the spaces $\ell_{p}^{\lambda}, c_{0}, c$ and $\ell_{\infty}$ overlap.

Further, the space $\ell_{p}^{\lambda}$ does not include the space $c_{0}$. To show this, we define the sequence $x=\left(x_{k}\right) \in c_{0}$ by

$$
x_{k}=\frac{1}{(k+1)^{1 / p}}, \quad k \in \mathbb{N} .
$$

Then, we have for every $n \in \mathbb{N}$ that

$$
\begin{gathered}
\left|\Lambda_{n}(x)\right|=\frac{1}{\lambda_{n}} \sum_{k=0}^{n} \frac{\lambda_{k}-\lambda_{k-1}}{(k+1)^{1 / p} \geq} \\
\geq \frac{1}{\lambda_{n}(n+1)^{1 / p}} \sum_{k=0}^{n}\left(\lambda_{k}-\lambda_{k}-1\right)=\frac{1}{(n+1)^{1 / p}}
\end{gathered}
$$

which shows that $\Lambda(x) \notin \ell_{p}$ and hence $x \notin \ell_{p}^{\lambda}$. Thus, the sequence $x$ is in $c_{0}$ but not in $\ell_{p}^{\lambda}$. Hence, the space $\ell_{p}^{\lambda}$ does not include any of the spaces $c_{0}, c$ or $\ell_{\infty}$.

Moreover, if $\lim \inf \frac{\lambda_{n+1}}{\lambda_{n}}=1$ then the space $\ell_{\infty}$ does not include the space $\ell_{p}^{\lambda}$. To see this, let $0<p<\infty$. Then, Lemma 3.4 implies that the sequence $y$, defined in the proof of Theorem 3.4, is in $\ell_{p}^{\lambda}$ but not in $\ell_{\infty}$. Therefore, none of the spaces $c_{0}, c$ or $\ell_{\infty}$ includes the space $\ell_{p}^{\lambda}$ when $\lim \inf \frac{\lambda_{n+1}}{\lambda_{n}}=1$, where $0<p<\infty$.

Corollary 3.2 is proved.

4. The basis for the space $\ell_{\boldsymbol{p}}^{\boldsymbol{\lambda}}$. In the present section, we give a sequence of points of the space $\ell_{p}^{\lambda}$ which forms a basis for $\ell_{p}^{\lambda}$, where $1 \leq p<\infty$.

If a normed sequence space $X$ contains a sequence $\left(b_{n}\right)$ with the property that for every $x \in X$ there is a unique sequence of scalars $\left(\alpha_{n}\right)$ such that

$$
\lim _{n}\left\|x-\left(\alpha_{0} b_{0}+\alpha_{1} b_{1}+\ldots+\alpha_{n} b_{n}\right)\right\|=0,
$$

then $\left(b_{n}\right)$ is called a Schauder basis (or briefly basis) for $X$. The series $\sum_{k} \alpha_{k} b_{k}$ which has the sum $x$ is then called the expansion of $x$ with respect to $\left(b_{n}\right)$, and written as $x=\sum_{k} \alpha_{k} b_{k}$. 
Now, because of the transformation $T$ defined from $\ell_{p}^{\lambda}$ to $\ell_{p}$ in the proof of Theorem 2.2 is onto, the inverse image of the basis $\left\{e^{(k)}\right\}_{k \in \mathbb{N}}$ of the space $\ell_{p}$ is the basis for the new space $\ell_{p}^{\lambda}$, where $1 \leq p<\infty$. Therefore, we have the following:

Theorem 4.1. Let $1 \leq p<\infty$ and define the sequence $e^{(k)}(\lambda)=\left\{e_{n}^{(k)}(\lambda)\right\}_{n \in \mathbb{N}}$ of the elements of the space $\ell_{p}^{\lambda}$ for every fixed $k \in \mathbb{N}$ by

$$
e_{n}^{(k)}(\lambda)=\left\{\begin{array}{ll}
(-1)^{n-k} \frac{\lambda_{k}}{\lambda_{n}-\lambda_{n-1}}, & k \leq n \leq k+1, \\
0, & n<k \text { or } n>k+1,
\end{array} \quad n \in \mathbb{N} .\right.
$$

Then, the sequence $\left\{e^{(k)}(\lambda)\right\}_{k \in \mathbb{N}}$ is a basis for the space $\ell_{p}^{\lambda}$ and every $x \in \ell_{p}^{\lambda}$ has a unique representation of the form

$$
x=\sum_{k} \alpha_{k}(\lambda) e^{(k)}(\lambda)
$$

where $\alpha_{k}(\lambda)=\Lambda_{k}(x)$ for all $k \in \mathbb{N}$,

Proof. Let $1 \leq p<\infty$. Then, it is clear by (4.1) that

$$
\Lambda\left(e^{(k)}(\lambda)\right)=e^{(k)} \in \ell_{p}, \quad k \in \mathbb{N},
$$

and hence $e^{(k)}(\lambda) \in \ell_{p}^{\lambda}$ for all $k \in \mathbb{N}$.

Further, let $x \in \ell_{p}^{\lambda}$ be given. For every non-negative integer $m$, we put

$$
x^{(m)}=\sum_{k=0}^{m} \alpha_{k}(\lambda) e^{(k)}(\lambda)
$$

Then, we have that

$$
\Lambda\left(x^{(m)}\right)=\sum_{k=0}^{m} \alpha_{k}(\lambda) \Lambda\left(e^{(k)}(\lambda)\right)=\sum_{k=0}^{m} \Lambda_{k}(x) e^{(k)}
$$

and hence

$$
\Lambda_{n}\left(x-x^{(m)}\right)=\left\{\begin{array}{ll}
0, & 0 \leq n \leq m, \\
\Lambda_{n}(x), & n>m,
\end{array} \quad n, m \in \mathbb{N} .\right.
$$

Now, for any given $\varepsilon>0$, there is a non-negative integer $m_{0}$ such that

$$
\sum_{n=m_{0}+1}^{\infty}\left|\Lambda_{n}(x)\right|^{p} \leq\left(\frac{\varepsilon}{2}\right)^{p}
$$

Hence, we have for every $m \geq m_{0}$ that

$$
\left\|x-x^{(m)}\right\|_{\ell_{p}^{\lambda}}=\left(\sum_{n=m+1}^{\infty}\left|\Lambda_{n}(x)\right|^{p}\right)^{1 / p} \leq\left(\sum_{n=m_{0}+1}^{\infty}\left|\Lambda_{n}(x)\right|^{p}\right)^{1 / p} \leq \frac{\varepsilon}{2}<\varepsilon .
$$

Thus, we obtain that 


$$
\lim _{m}\left\|x-x^{(m)}\right\|_{\ell_{p}^{\lambda}}=0
$$

which shows that $x \in \ell_{p}^{\lambda}$ is represented as in (4.2).

Finally, let us show the uniqueness of the representation (4.2) of $x \in \ell_{p}^{\lambda}$. For this, suppose on the contrary that there exists another representation $x=\sum_{k} \beta_{k}(\lambda) e^{(k)}(\lambda)$. Since the linear transformation $T$ defined from $\ell_{p}^{\lambda}$ to $\ell_{p}$ in the proof of Theorem 2.2 is continuous [19] (Theorem 4.2.8), we have that

$$
\Lambda_{n}(x)=\sum_{k} \beta_{k}(\lambda) \Lambda_{n}\left(e^{(k)}(\lambda)\right)=\sum_{k} \beta_{k}(\lambda) e_{n}^{(k)}=\beta_{n}(\lambda), \quad n \in \mathbb{N},
$$

which contradicts the fact that $\Lambda_{n}(x)=\alpha_{n}(\lambda)$ for all $n \in \mathbb{N}$. Hence, the representation (4.2) of $x \in \ell_{p}^{\lambda}$ is unique.

Theorem 4.1 is proved.

Now, it is known by Theorem 2.1 that $\ell_{p}^{\lambda}, 1 \leq p<\infty$, is a Banach space with its natural norm. This leads us together with Theorem 4.1 to the following corollary:

Corollary 4.1. The sequence space $\ell_{p}^{\lambda}$ of non-absolute type is separable for $1 \leq$ $\leq p<\infty$.

Finally, we conclude our work by expressing from now on that the aim of the next paper is to determine the $\alpha$-, $\beta$ - and $\gamma$-duals of the space $\ell_{p}^{\lambda}$ and is to characterize some matrix classes concerning the space $\ell_{p}^{\lambda}$, where $1 \leq p<\infty$.

1. Altay B., Başar F. Some Euler sequence spaces of non-absolute type // Ukr. Math. J. - 2005. - 57, № 1 . - P. $1-17$.

2. Altay B., Bașar F., Mursaleen M. On the Euler sequence spaces which include the spaces $\ell_{p}$ and $\ell_{\infty}$ I // Inform. Sci. - 2006. - 176, № 10. - P. 1450-1462.

3. Aydın C., Başar F. On the new sequence spaces which include the spaces $c_{0}$ and $c / /$ Hokkaido Math. J. - 2004. - 33, № 2. - P. $383-398$.

4. Aydın C., Başar F. Some new paranormed sequence spaces // Inform. Sci. - 2004. - 160, № 1-4. P. $27-40$.

5. Aydın C., Başar F. Some new difference sequence spaces // Appl. Math. and Comput. - 2004. - 157, № 3. - P. $677-693$.

6. Aydin C., Başar F. Some new sequence spaces which include the spaces $\ell_{p}$ and $\ell_{\infty} / /$ Demonstr. math. - 2005. - 38, № 3. - P. $641-656$.

7. Başar F., Altay B. On the space of sequences of $p$-bounded variation and related matrix mappings // $\mathrm{Ukr}$ Math. J. - 2003. - 55, № 1. - P. 136-147.

8. Hardy G. H., Littlewood J. E., Polya G. Inequalities. - Cambridge Univ. Press, 1952.

9. Krěn S. G., Petunin Ju. I., Semenov E. M. Interpolation of linear operators. - Providence, Rhode Island: Amer. Math. Soc.,1982.

10. Maddox I. J. Elements of functional analysis. - 2nd ed. - Cambridge: Cambridge Univ. Press, 1988.

11. Malkowsky E. Recent results in the theory of matrix transformations in sequence spaces // Mat. Vesnik. - 1997. - 49. - P. 187-196.

12. Malkowsky E., Savaş E. Matrix transformations between sequence spaces of generalized weighted means // Appl. Math. and Comput. - 2004. - 147, № 2. - P. 333 - 345.

13. Mursaleen M., Başar F., Altay B. On the Euler sequence spaces which include the spaces $\ell_{p}$ and $\ell_{\infty}$ II // Nonlinear Analysis: TMA. - 2006. - 65, № 3. - P. 707 - 717.

14. Mursaleen M., Noman A. K. On the spaces of $\lambda$-convergent and bounded sequences // Thai J. Math. 2010. - 8, № 2. - P. $311-329$.

15. Mursaleen M., Noman A. K. On som new differnce sequence spaces of non-absolute type // Math Comput. Mod. - 2010. - 52. - P. 603-617.

16. $N g$ P.-N. On modular space of a nonabsolute type // Nanta Math. - 1978. - 2. - P. $84-93$.

17. Ng P.-N., Lee P.-Y. Cesàro sequence spaces of non-absolute type // Comment. Math. Prace Mat. - 1978 - 20, № 2. - P. 429-433.

18. Wang C.-S. On Nörlund sequence spaces // Tamkang J. Math. - 1978. - 9. - P. 269-274.

19. Wilansky A. Summability through functional analysis // North-Holland Math. Stud. - Amsterdam etc. 1984. $\mathbf{- 8 5}$. 